Supporting Information for

\title{
Defect-Induced Inhomogeneous Phase Transition in 2D Perovskite Single Crystals at Low Temperatures
}

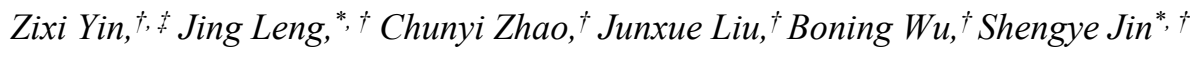

†State Key Laboratory of Molecular Reaction Dynamics and Dynamics Research Center for Energy and Environmental Materials, Dalian Institute of Chemical Physics, Chinese Academy of Sciences, Dalian 116023, China

\#niversity of Chinese Academy of Sciences, Beijing 100049, China

\section{Synthesis of $\left(\mathrm{n}-\mathrm{C}_{4} \mathrm{H}_{9} \mathrm{NH}_{3}\right)_{2} \mathrm{PbI}_{4}$ single crystals}

$\left(\mathrm{n}-\mathrm{C}_{4} \mathrm{H}_{9} \mathrm{NH}_{3}\right)_{2} \mathrm{PbI}_{4} \mathrm{SCs}$ were synthesized and purified by moderate liquid method based on previous report. ${ }^{1}$ The materials used in the experiments were purchased from Sigma-Aldrich and used without further processing.

$\mathrm{PbO}$ powder $(2.232 \mathrm{~g}), 57 \% \mathrm{w} / \mathrm{w} \mathrm{HI}$ (water solution, $10 \mathrm{~mL}$ ) and $50 \% \mathrm{w} / \mathrm{w}$ $\mathrm{H}_{3} \mathrm{PO}_{2}$ in water $(1.7 \mathrm{~mL})$ were mixed, and kept heating under constant stirring until $\mathrm{PbO}$ was completely dissolved. $5 \mathrm{~mL} \mathrm{HI}$ was also added dropwise into $924 \mu \mathrm{L}$ n-Butylamine in an ice bath to make a n- $\mathrm{C}_{4} \mathrm{H}_{9} \mathrm{NH}_{3} \mathrm{I}$ solution. Then, the $\mathrm{n}-\mathrm{C}_{4} \mathrm{H}_{9} \mathrm{NH}_{3} \mathrm{I}$ solution was dropped into the hot mixture under magnetic stirring. The combined solution was cooled to room temperature, allowing layered orange crystals to grow. The crystals were rinsed with anhydrous ether and extracted by suction filtration, before dried at $60{ }^{\circ} \mathrm{C}$ for $24 \mathrm{~h}$ under reduced pressure.

High-quality single crystals were formed through a recrystallized process from the orange raw crystals: $8.5 \mathrm{~mL} \mathrm{H}_{3} \mathrm{PO}_{2}$ and $5 \mathrm{~mL} \mathrm{HI}$ were mixed under stirring. The 
well-dried flake crystals were dissolved in the $\mathrm{HI} / \mathrm{H}_{3} \mathrm{PO}_{2}$ solution, and then the mixture was saturated by vaporizing the solvent at a fixed temperature of $70{ }^{\circ} \mathrm{C}$. The grown crystals were again washed by anhydrous ether, filtered out, and dried at $60{ }^{\circ} \mathrm{C}$ for $24 \mathrm{~h}$ under reduced pressure.

\section{Photoluminescence (PL)-scanned imaging microscope experiment}

We used a home-built photoluminescence (PL)-scanned imaging microscope coupled with a time-correlated single photon counting (TCSPC) module to map the PL kinetics on an individual crystal. The excitation beam is a picosecond pulse diode laser with $405 \mathrm{~nm}$ output wavelength $(20 \mathrm{MHz}$ or $0.5 \mathrm{MHz}$ repetition rate). For wide-field illumination, the laser beam is defocused before a $100 \times$ air objective lens $(\mathrm{NA}=0.95)$ to form an excitation spot of $\sim 60 \mu \mathrm{m}$ in diameter, which ensures the single crystal is excited homogeneously. A lateral scan of PL on the sample was achieved by rotating a galvanometer mirror before the detector. Each scanning image contains $256 \times 256$ pixels, The PL signal was collected using a high-speed detector (HPM-100-50, Hamamatsu, Japan) with a $550 \mathrm{~nm}$ long pass filter, $480 \pm 10 \mathrm{~nm}$ band pass filter, $525 \pm 20 \mathrm{~nm}$ band pass filter or $510 \mathrm{~nm}$ long pass filter and $540 \mathrm{~nm}$ short pass filter.

\section{Ultrafast transient absorption (TA) Measurement}

The femtosecond TA setup is based on a regenerative amplified Ti:sapphire laser system from Coherent $(800 \mathrm{~nm}, 35 \mathrm{fs}, 6 \mathrm{~mJ}$ pulse-1, and $1 \mathrm{kHz}$ repetition rate), nonlinear frequency mixing techniques and the Femto-TA100 spectrometer 
(Time-Tech Spectra). Briefly, the $800 \mathrm{~nm}$ output pulse from the regenerative amplifier was split into two beams by a $50 \%$ beam splitter. The transmitted part was used to pump a $\beta-\mathrm{BaB}_{2} \mathrm{O}_{4}$ crystal (BBO) to generate a $400 \mathrm{~nm}$ output, which is selected as pump beam due to its higher power stability than TOPAS OPA output. The reflected $800 \mathrm{~nm}$ beam was split again into two parts. One part with less than $10 \%$ was attenuated with an OD filter and focused into a $2 \mathrm{~mm}$ thick sapphire window to generate a white light continuum (WLC) from $400 \mathrm{~nm}$ to $800 \mathrm{~nm}$ used as probe beam. The probe beam was focused with an Al parabolic reflector onto the sample. After the sample, the probe beam was collimated and then focused into a fiber-coupled spectrometer with CMOS sensors and detected at a frequency of $1 \mathrm{kHz}$. The delay between the pump and probe pulses was controlled by a motorized delay stage. The pump pulses were chopped by a synchronized chopper at $500 \mathrm{~Hz}$ and the absorbance change was calculated with two adjacent probe pulses (pump-blocked and pump-unblocked).

\section{Structure characterization}

Transmission electron microscopy (TEM) and selected area electron diffraction (SAED) images were obtained on JEM-2100 microscope. X-ray diffraction (XRD) patterns were obtained on a X'pert Pro X-Ray Diffractometer (PANAlytical, Netherlands) using $\mathrm{Cu} \mathrm{Ka}$ radiation.

\section{Definition of the degree of phase transition in 2D perovskite crystals}

Normally, the degree of phase transition (here denoted as $x$ ) from the RT phase 
to the LT phase in $2 \mathrm{D}$ perovskite single crystals can be described by the ratio of the amounts between LT phase at low temperatures and RT phase at $293 \mathrm{~K}$ as follows.

$$
x=\frac{n_{L T}}{n_{R T}}
$$

where $n_{L T}$ and $n_{R T}$ represent the amount of LT phase at low temperatures and RT phase at $293 \mathrm{~K}$ respectively. For 2D perovskites, the PL lifetime is mainly determined by nonradiative process due to the existence of lots of defects; therefore, their PL intensity should be proportional to the product of their amount and PL lifetime as:

$$
\begin{gathered}
I_{L T} \propto n_{L T} \tau_{L T} \\
I_{R T} \propto n_{R T} \tau_{R T}
\end{gathered}
$$

where $I_{L T}$ and $I_{R T}$ are the PL intensity of the LT phase and the RT phase respectively, and $\tau_{L T}$ and $\tau_{R T}$ represent the PL lifetime of LT phase and RT phase. Therefore, the degree of phase transition $(x)$ can be derived according to Eq. (S1) as follows:

$$
x \propto \frac{I_{L T}}{I_{R T}} /{ }_{\frac{\tau_{L T}}{\tau_{R T}}} \propto \frac{I_{L T / R T}}{\tau_{L T / R T}}
$$

where $I_{L T / R T}\left(\tau_{L T / R T}\right)$ represent the ratio of the intensity (lifetime) between LT-phase PL at $83 \mathrm{~K}$ and RT-phase PL at $293 \mathrm{~K}$.

\section{REFERENCES}

(1) Stoumpos, C. C.; Cao, D. H.; Clark, D. J.; Young, J.; Rondinelli, J. M.; Jang, J. I.; Hupp, J. T.; Kanatzidis, M. G., Ruddlesden-Popper Hybrid Lead Iodide Perovskite 2D Homologous Semiconductors. Chem. Mater. 2016, 28, 2852-2867. 
$\mathbf{a}$

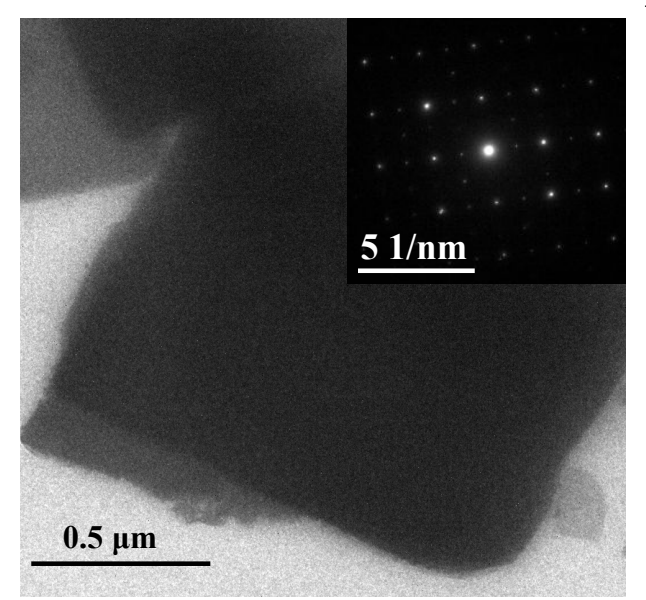

b

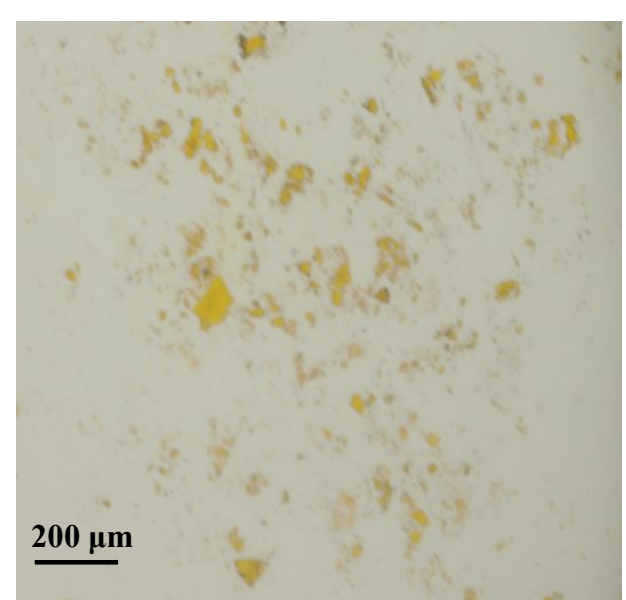

Figure S1. (a) TEM image and selected area electron diffraction (SAED) image (inset) of $\left(\mathrm{n}-\mathrm{C}_{4} \mathrm{H}_{9} \mathrm{NH}_{3}\right)_{2} \mathrm{PbI}_{4}$ crystal; (b) The visible image of a bunch of $\left(\mathrm{n}-\mathrm{C}_{4} \mathrm{H}_{9} \mathrm{NH}_{3}\right)_{2} \mathrm{PbI}_{4}$ crystals at $293 \mathrm{~K}$.
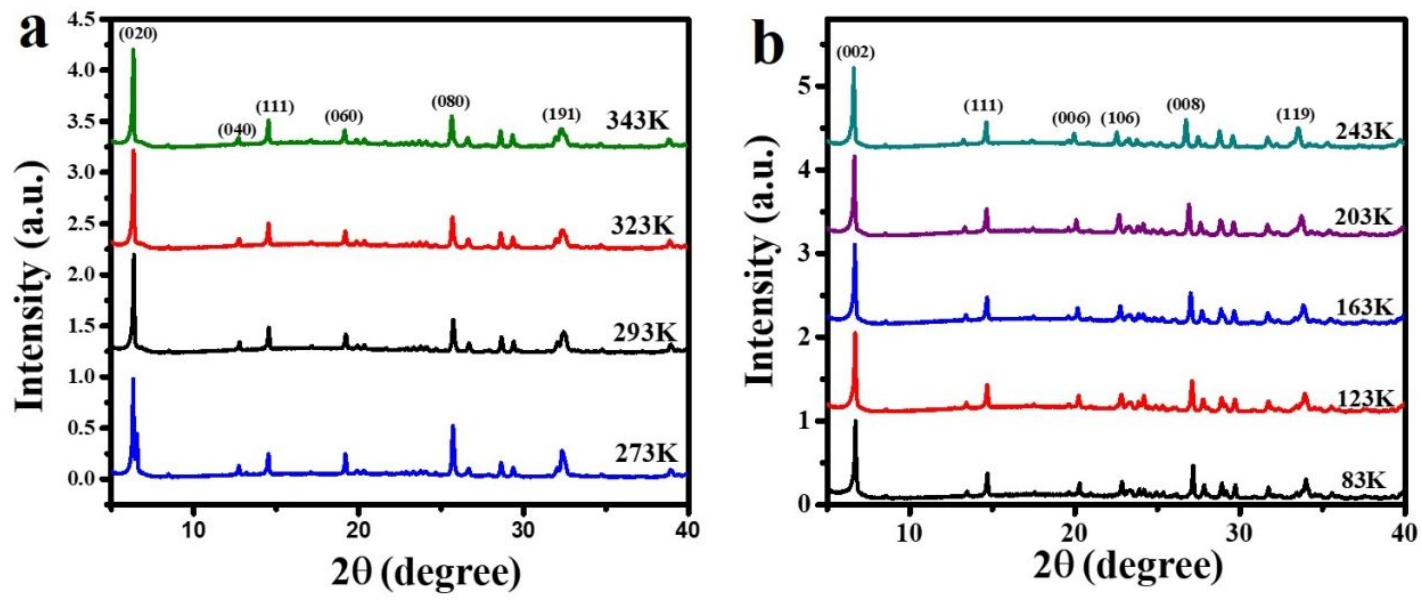

Figure S2. Temperature-dependent XRD patterns of single crystals above $273 \mathrm{~K}$ (a) and below $243 \mathrm{~K}(\mathrm{~b})$. 

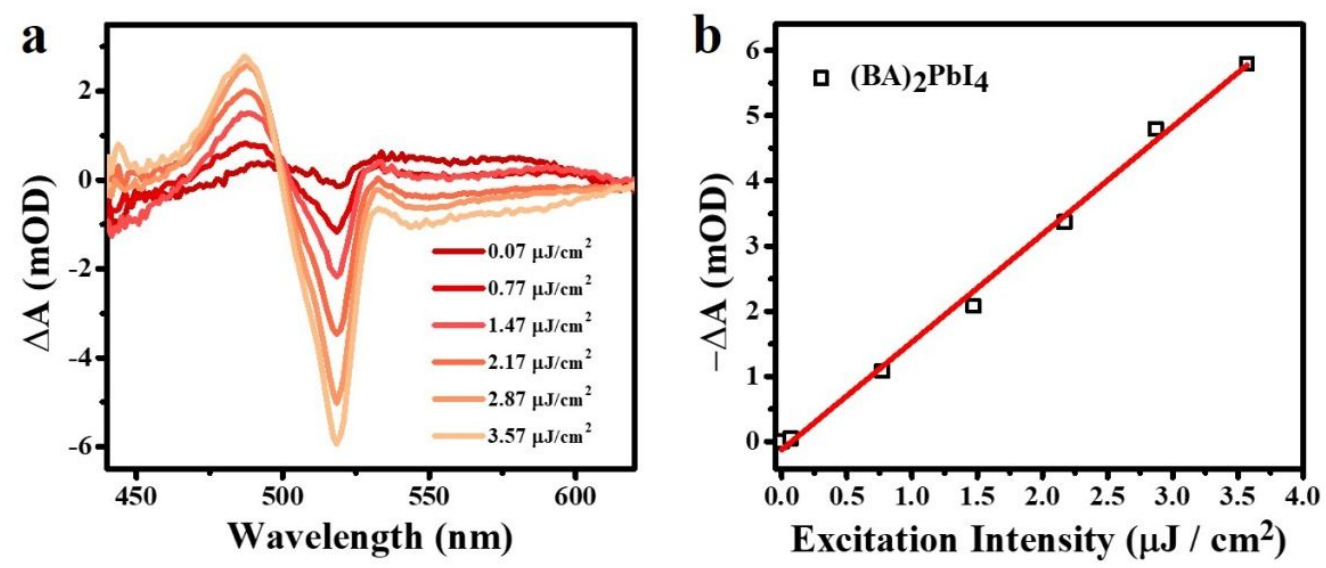

Figure S3. (a) TA spectra of $\left(n-C_{4} \mathrm{H}_{9} \mathrm{NH}_{3}\right)_{2} \mathrm{PbI}_{4} \mathrm{SCs}$ at delay of 2 ps under the excitation at $400 \mathrm{~nm}$ with indicated intensities. (b) The plot of the peak intensity of TA spectra $(-\triangle \mathrm{A})$ recorded at $517 \mathrm{~nm}$ as a function of excitation intensity, indicating that the transient absorption response is linear for the pump intensity less than 3.5 $\mu \mathrm{J} / \mathrm{cm}^{2}$. 

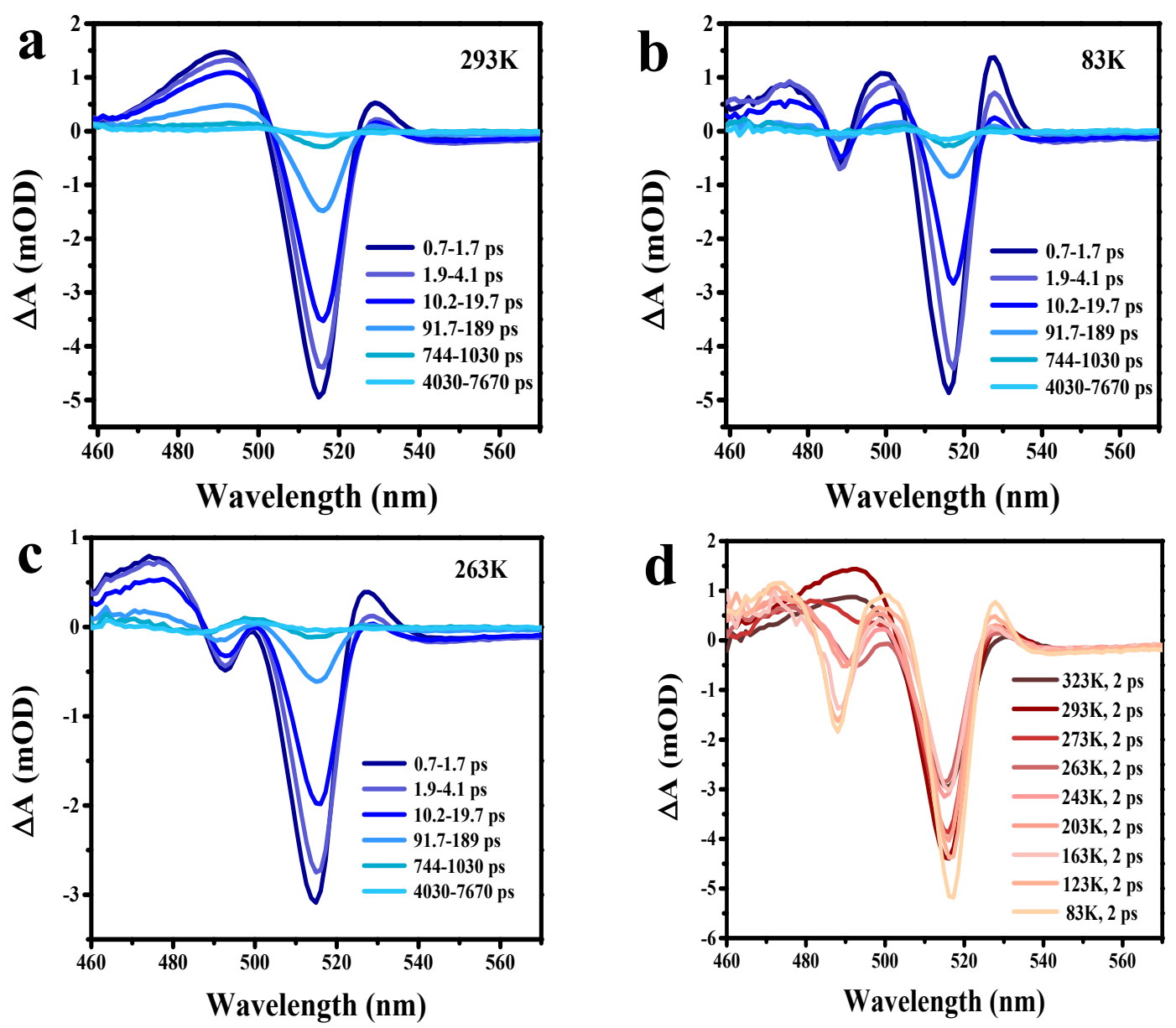

Figure S4. TA spectra of $\left(\mathrm{n}-\mathrm{C}_{4} \mathrm{H}_{9} \mathrm{NH}_{3}\right)_{2} \mathrm{PbI}_{4} \mathrm{SCs}$ at indicated delays with $400 \mathrm{~nm}$ excitation probed respectively at (a) $\mathrm{T}=293 \mathrm{~K}$, (b) $\mathrm{T}=83 \mathrm{~K}$ and (c) $\mathrm{T}=263 \mathrm{~K}$; (d) The comparison of TA spectra of $\left(n-C_{4} \mathrm{H}_{9} \mathrm{NH}_{3}\right)_{2} \mathrm{PbI}_{4} \mathrm{SCs}$ at fixed delay $(\sim 2 \mathrm{ps})$ measured at different temperatures. 

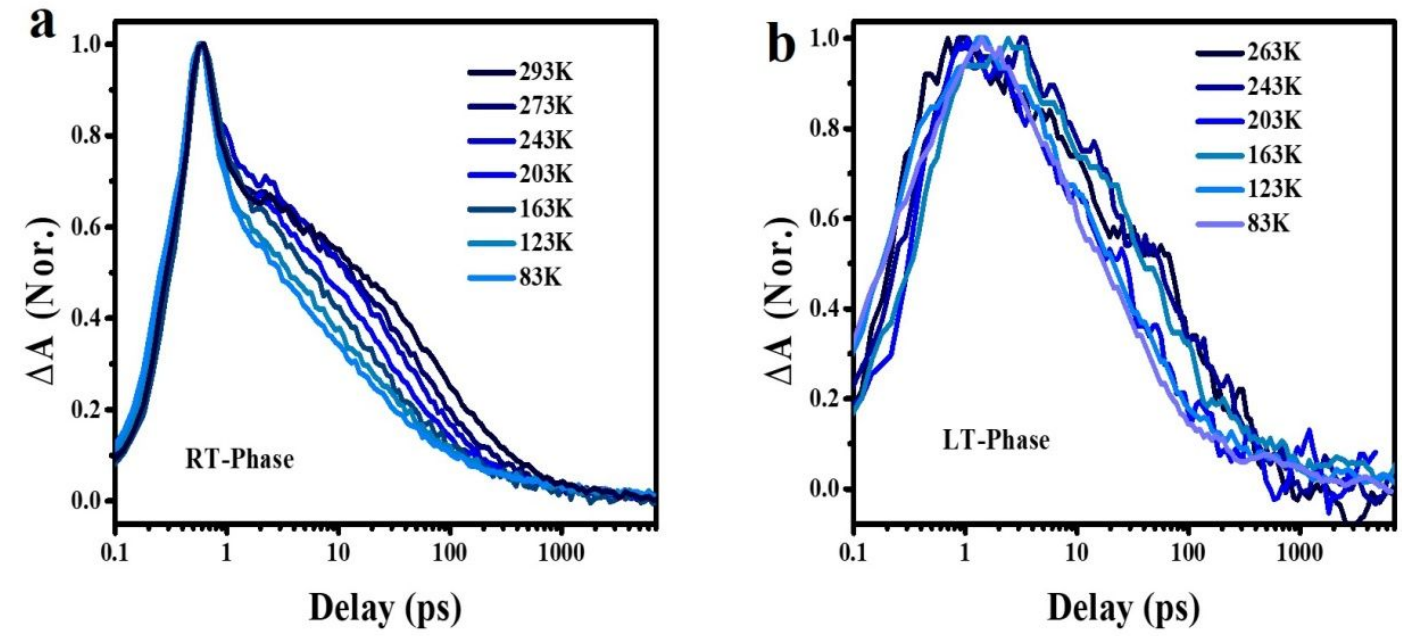

Figure S5. Normalized TA bleach kinetics respectively from (a) RT-phase (probed at $517 \mathrm{~nm}$ ) and (b) LT-phase (probed at $489 \mathrm{~nm})$ excitons in $\left(\mathrm{n}-\mathrm{C}_{4} \mathrm{H}_{9} \mathrm{NH}_{3}\right)_{2} \mathrm{PbI}_{4} \mathrm{SCs}$ at different temperatures.
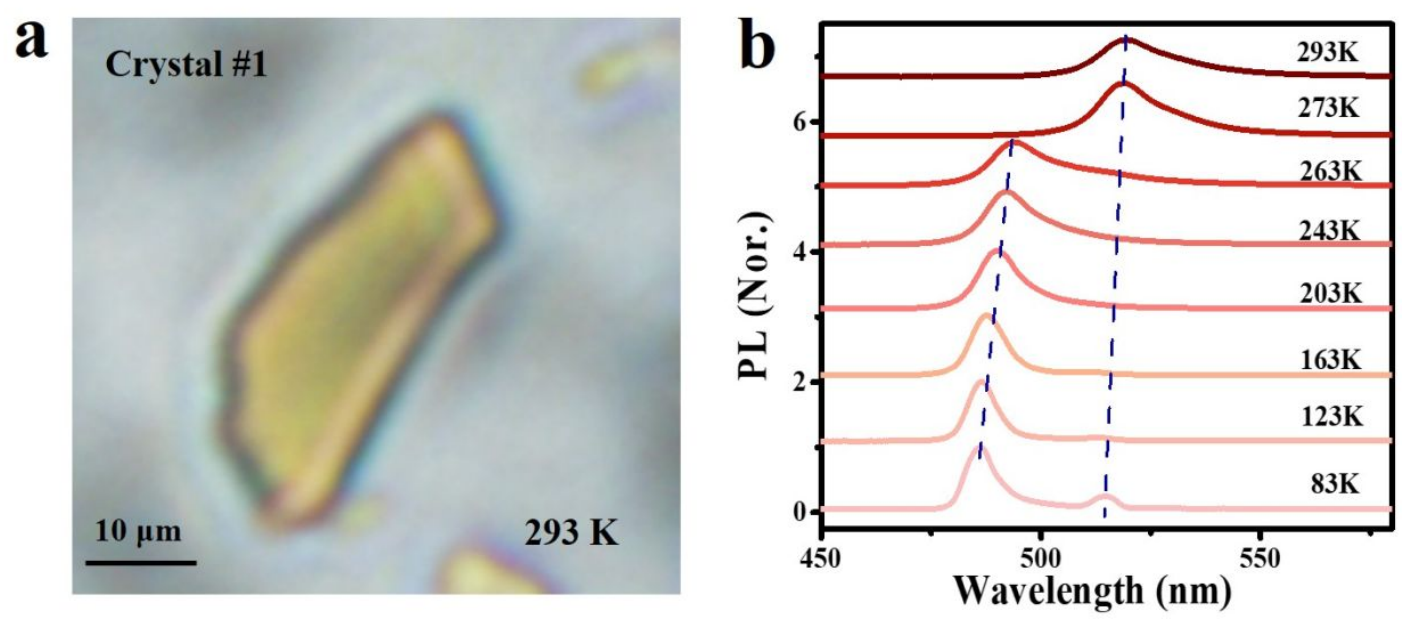

Figure S6. (a) The visible image of $\left(n-\mathrm{C}_{4} \mathrm{H}_{9} \mathrm{NH}_{3}\right)_{2} \mathrm{PbI}_{4} \mathrm{SC}$ (crystal \#1) used in the main text at $293 \mathrm{~K}$; (b) The PL spectra of $\left(\mathrm{n}-\mathrm{C}_{4} \mathrm{H}_{9} \mathrm{NH}_{3}\right)_{2} \mathrm{PbI}_{4} \mathrm{SC}$ (crystal \#1) from 83 K to $293 \mathrm{~K}$. 

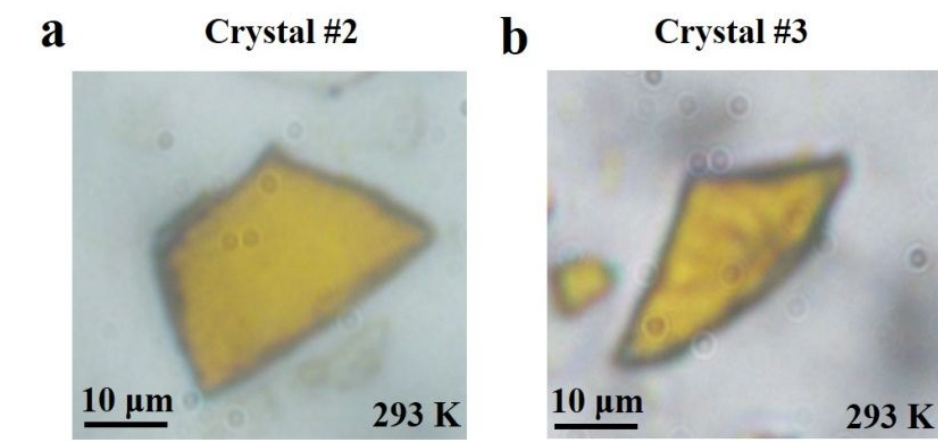

c Crystal \#4
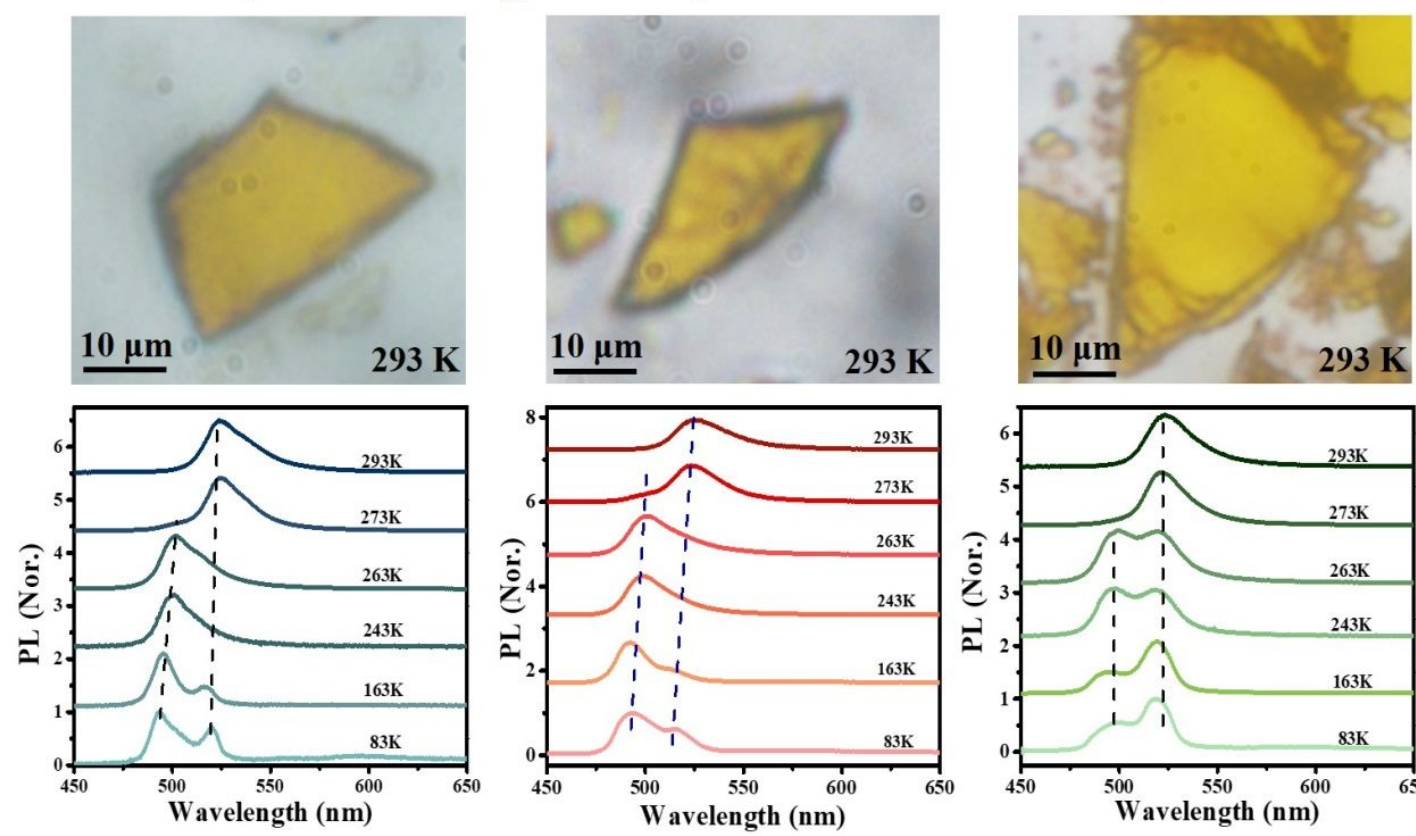

Figure S7. Temperature-dependent PL spectra from (a) Crystal \#2, (b) Crystal \#3 and

(c) Crystal \#4 collected under various temperatures from $83 \mathrm{~K}$ to $293 \mathrm{~K}$. The upper panels are their optical images at $293 \mathrm{~K}$. 

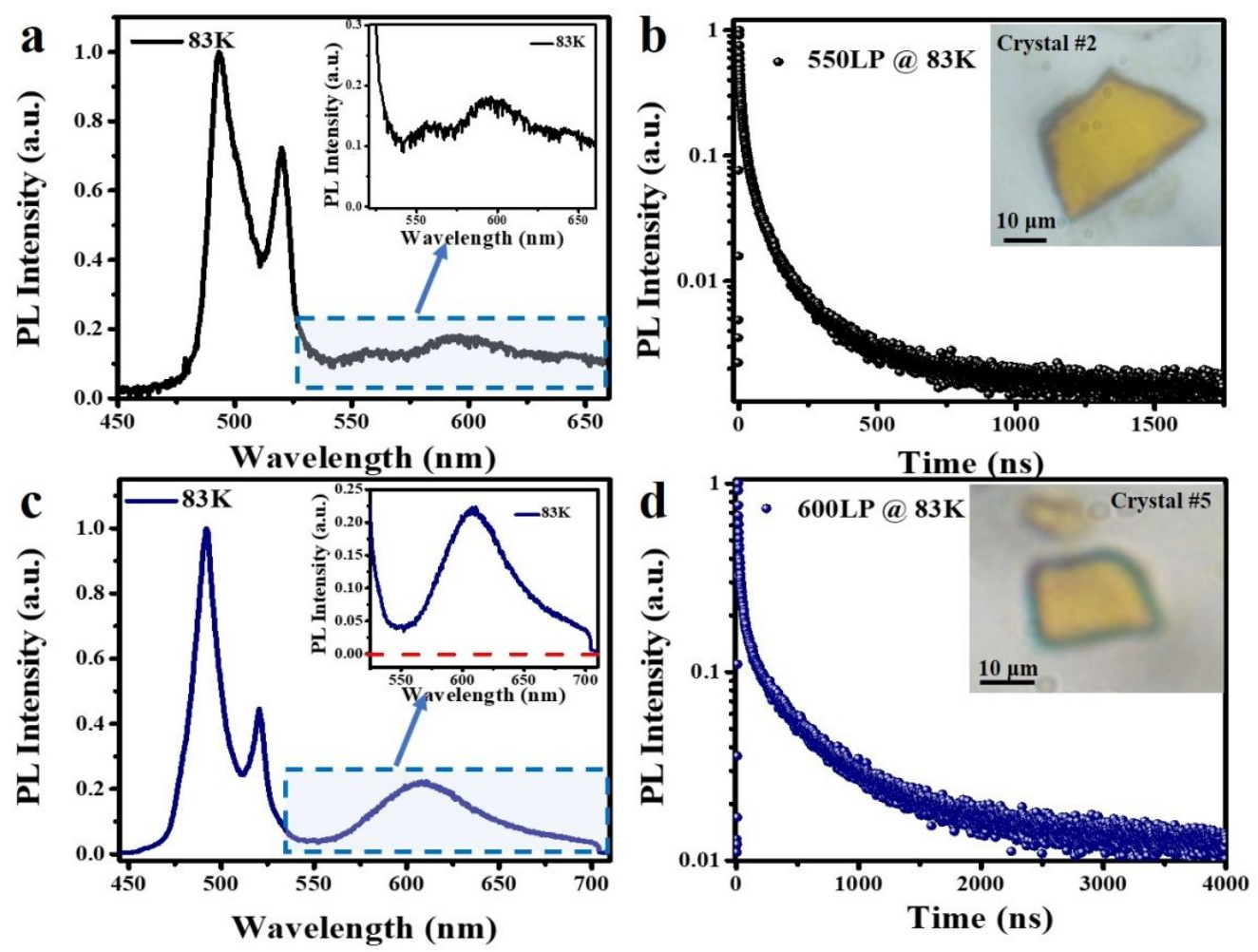

Figure S8. The measured PL spectra and kinetics of broadband emission from crystal $\# 2$ (a, b) and crystal \#5 (c, d) at $83 \mathrm{~K}$. The insets in panel b and d are their optical images at $293 \mathrm{~K}$. 


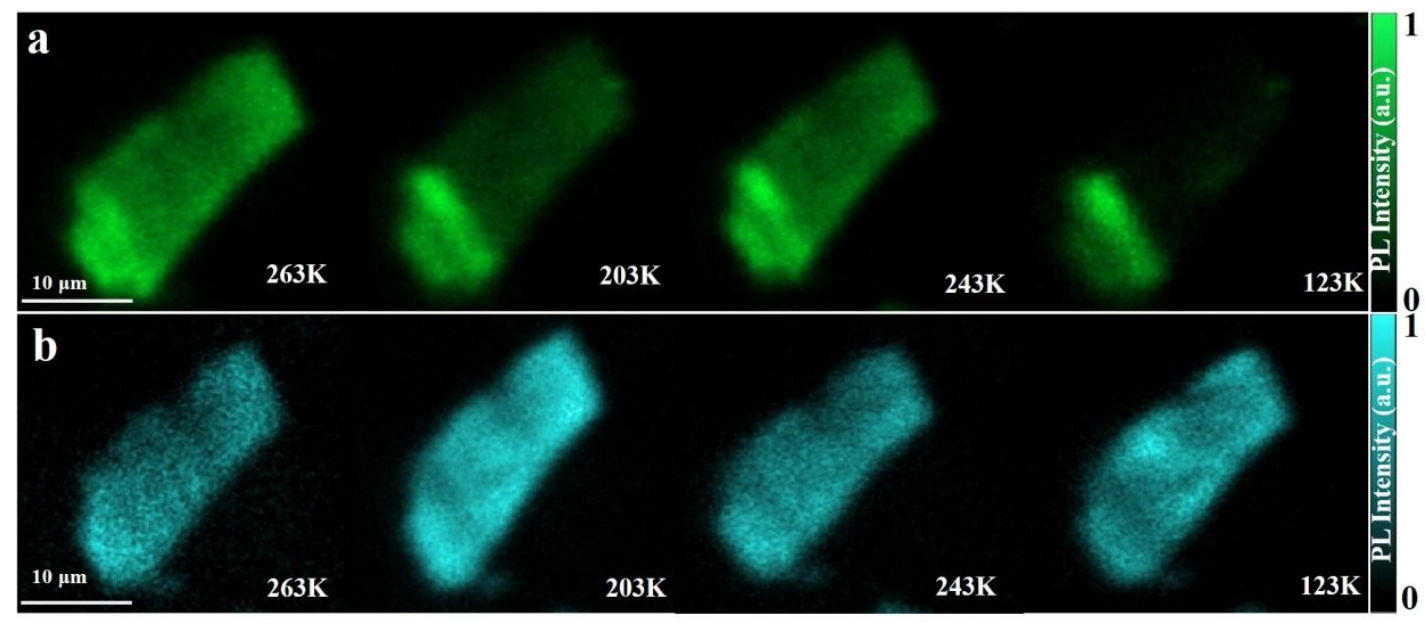

Figure S9. The PL intensity images from RT phase (a) and LT phase (b) in an individual ( $\left(\mathrm{n}-\mathrm{C}_{4} \mathrm{H}_{9} \mathrm{NH}_{3}\right)_{2} \mathrm{PbI}_{4} \mathrm{SC}$ (i.e., crystal \#1 used in the main text) measured at other low temperatures. 


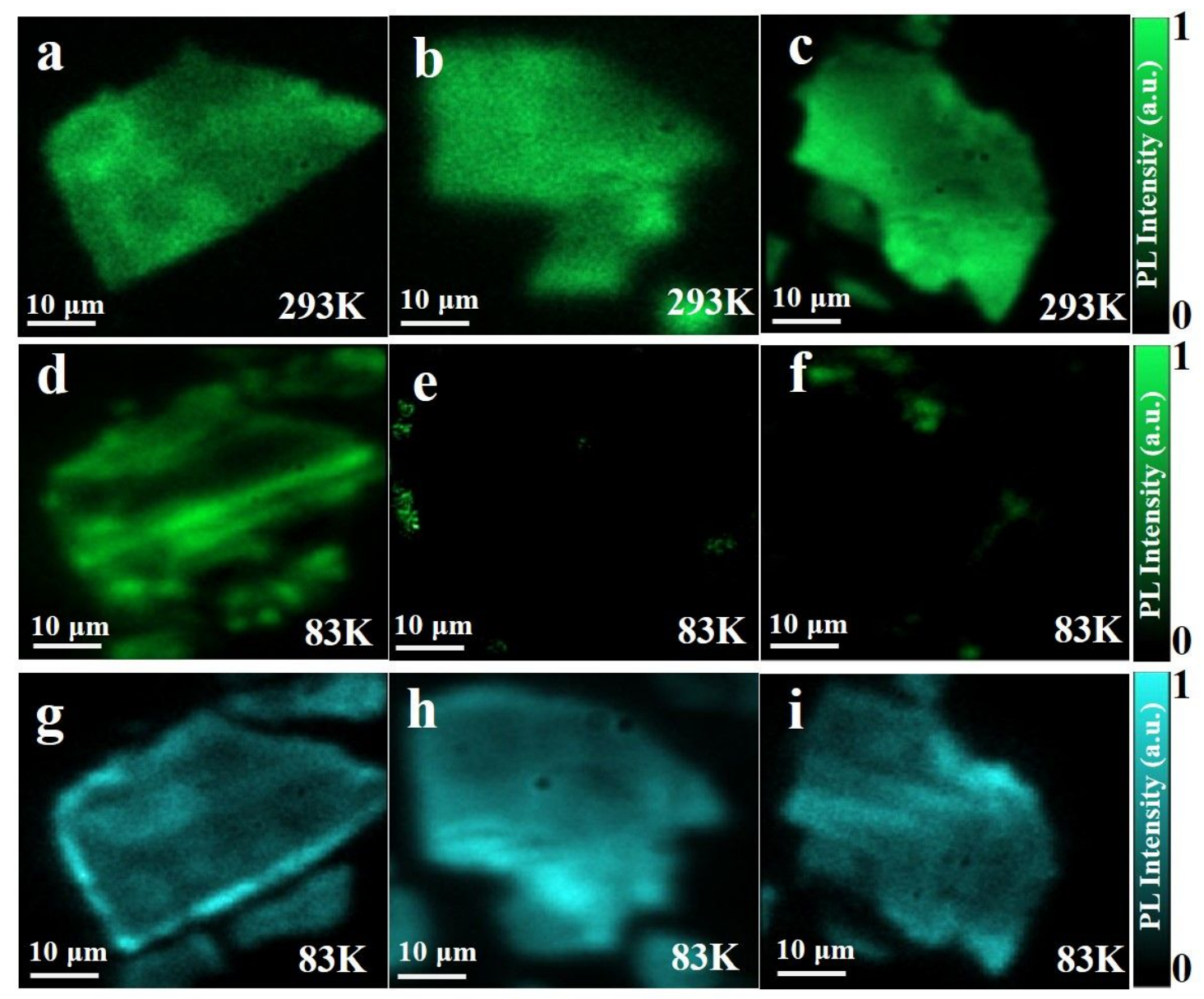

Figure S10. The PL intensity images from RT phase recorded at $293 \mathrm{~K}(\mathrm{a}-\mathrm{c})$ and at $83 \mathrm{~K}(\mathrm{~d}-\mathrm{f})$ respectively, as well as from LT phase at $83 \mathrm{~K}(\mathrm{~g}-\mathrm{i})$ in three different $\left(\mathrm{n}-\mathrm{C}_{4} \mathrm{H}_{9} \mathrm{NH}_{3}\right)_{2} \mathrm{PbI}_{4} \mathrm{SCs}$. Similar to the crystal in main text, the PL images from two different phases at $83 \mathrm{~K}$ in these SCs also show inhomogeneous but roughly complementary distribution trend. 

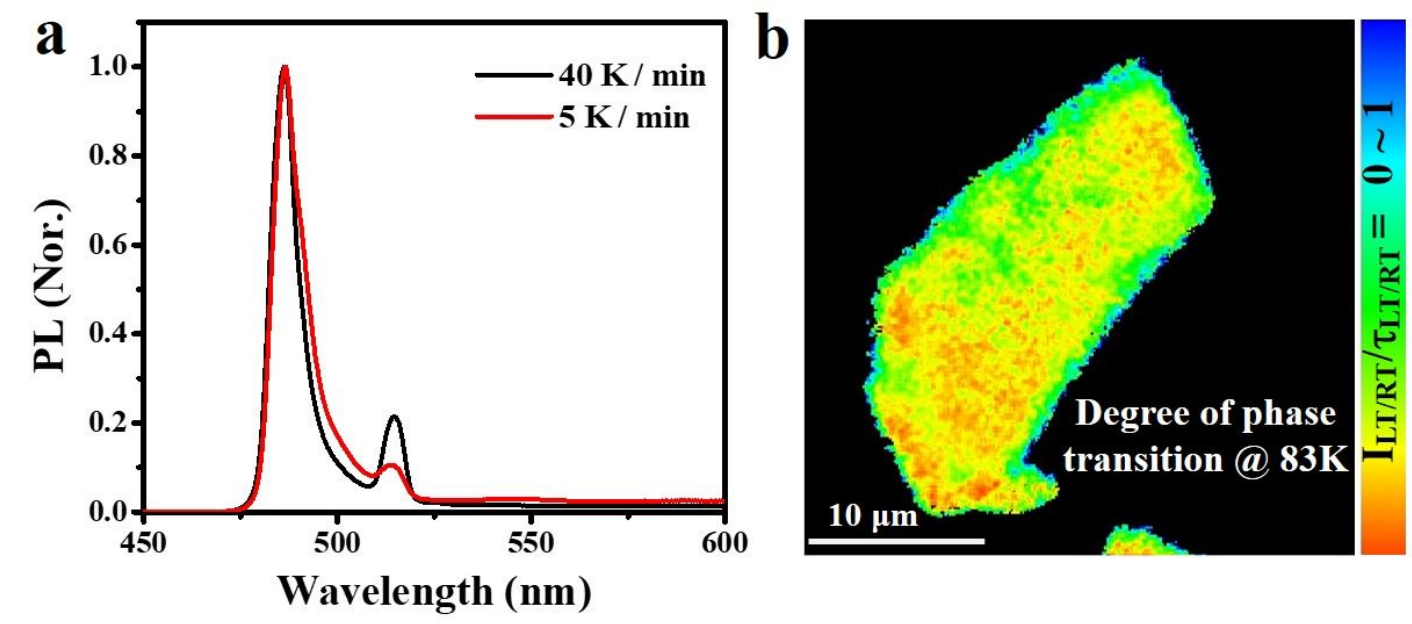

Figure S11. (a) The comparison of the PL spectra from the $\left(n-\mathrm{C}_{4} \mathrm{H}_{9} \mathrm{NH}_{3}\right)_{2} \mathrm{PbI}_{4} \mathrm{SC}$ (crystal \#1) at $83 \mathrm{~K}$ under $405 \mathrm{~nm}$ excitation with different cooling rates, implying that the slower cooling rate leads to the higher degree of phase transition. (b) The map of the normalized degree of phase transition at $83 \mathrm{~K}$ under a slower cooling rate of 5 $\mathrm{K} / \mathrm{min}$, which also exhibits an inhomogeneous distribution with a slightly higher degree of phase transition and less phase inhomogeneity, compared to those under the faster cooling rate of $40 \mathrm{~K} / \mathrm{min}$ (see Figure $3 \mathrm{~b}$ in main text). 
a
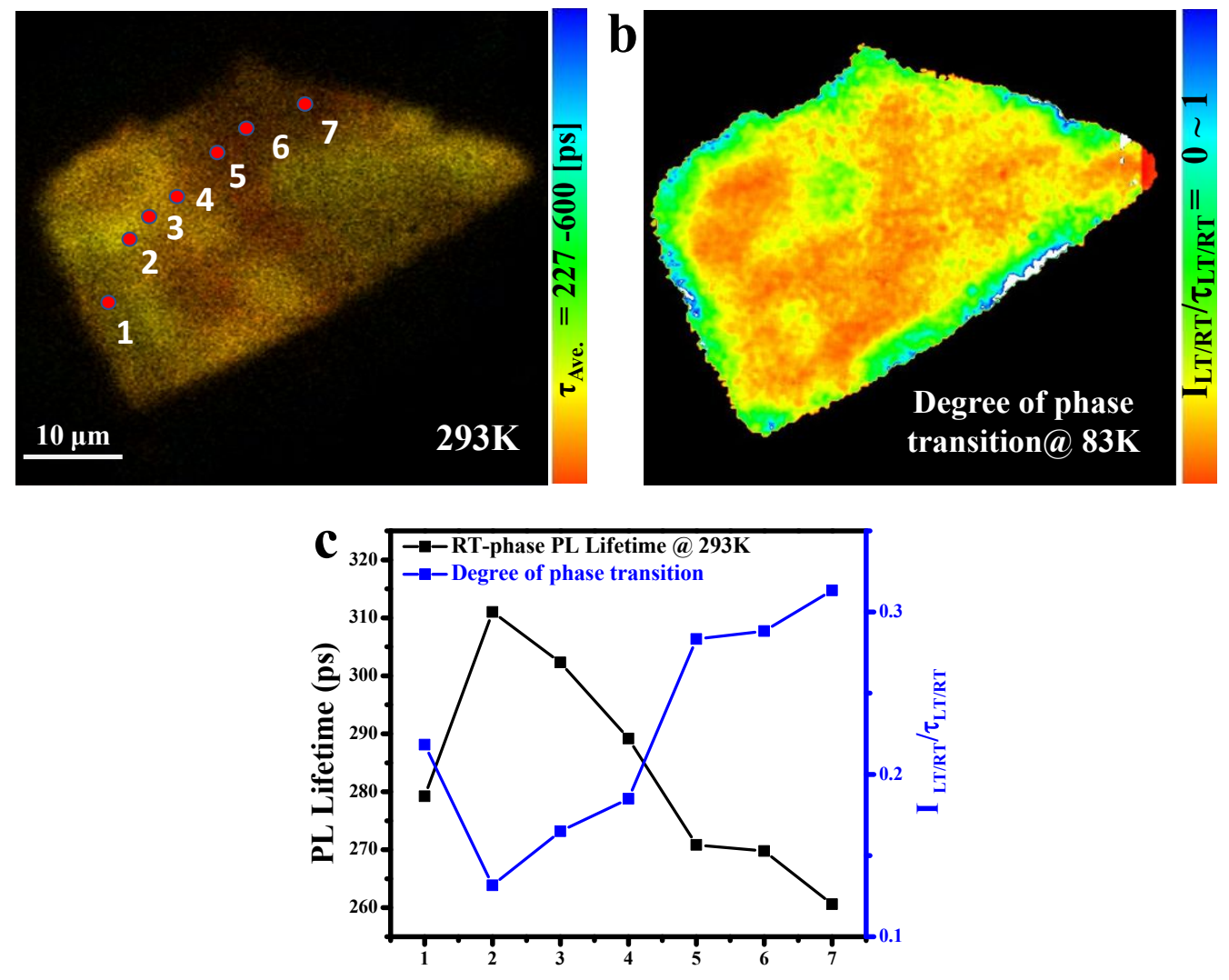

Position No.

Figure S12. (a) The RT-phase PL lifetime image measured in an individual $\left(\mathrm{n}-\mathrm{C}_{4} \mathrm{H}_{9} \mathrm{NH}_{3}\right)_{2} \mathrm{PbI}_{4} \mathrm{SC}$ at $293 \mathrm{~K}$. (b) The distribution map of the relative completion degree of phase transition at $83 \mathrm{~K}$ calculated according to Eq.(S4), showing a rough inverse correlation relative to the RT-phase PL lifetime image shown in panel a. (c) The comparison of the RT-phase PL lifetimes and the degrees of phase transition from panel $b$ at various positions (denoted by red points) as shown in panel a. 
$\mathbf{a}$
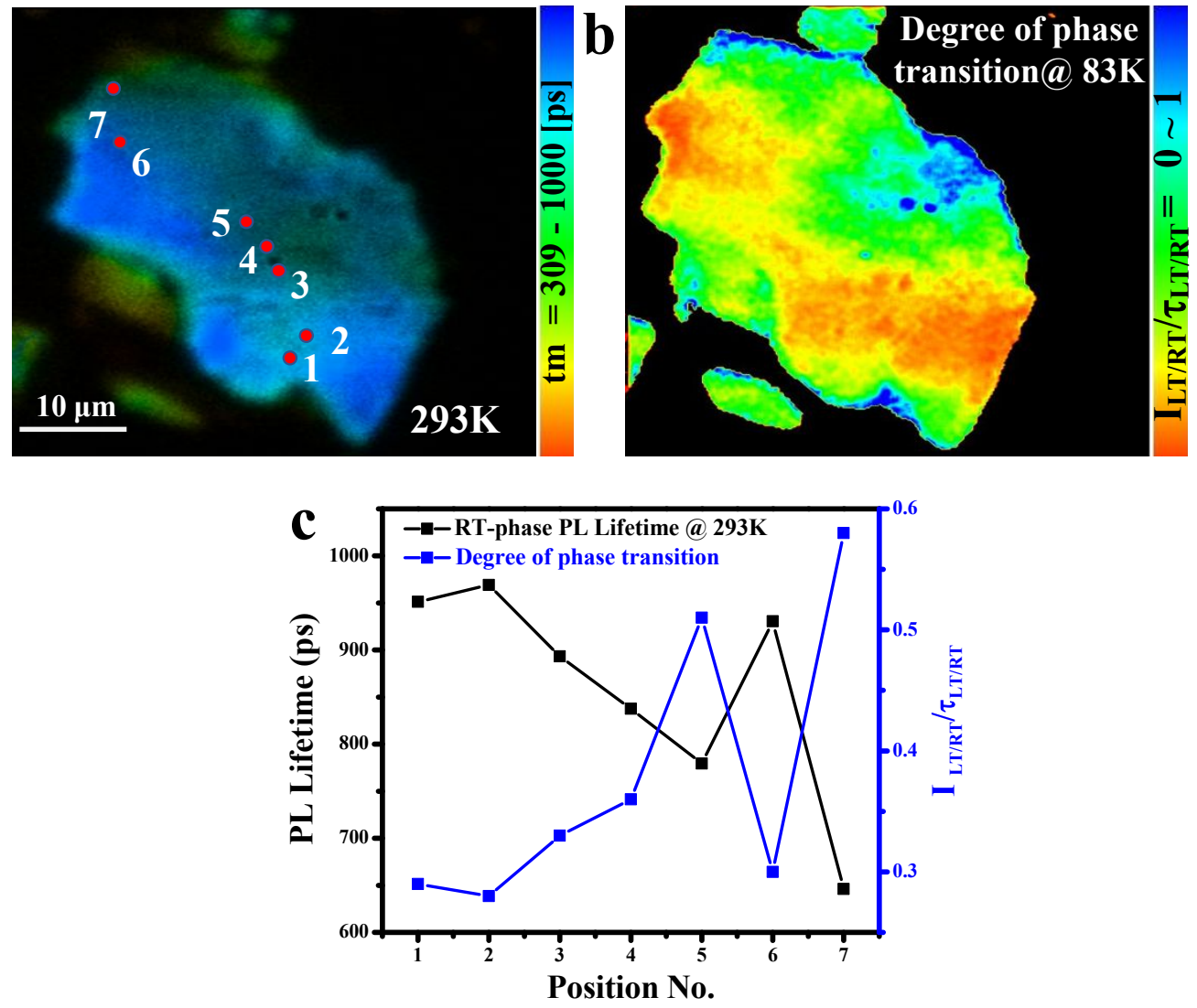

Figure S13. (a) The RT-phase PL lifetime image measured in an individual $\left(\mathrm{n}-\mathrm{C}_{4} \mathrm{H}_{9} \mathrm{NH}_{3}\right)_{2} \mathrm{PbI}_{4} \mathrm{SC}$ at $293 \mathrm{~K}$. (b) The distribution map of the relative completion degree of phase transition at $83 \mathrm{~K}$ calculated according to Eq.(S4), showing a rough inverse correlation relative to the RT-phase PL lifetime image shown in panel a. (c) The comparison of the RT-phase PL lifetimes and the degrees of phase transition from panel $b$ at various positions (denoted by red points) as shown in panel a. 

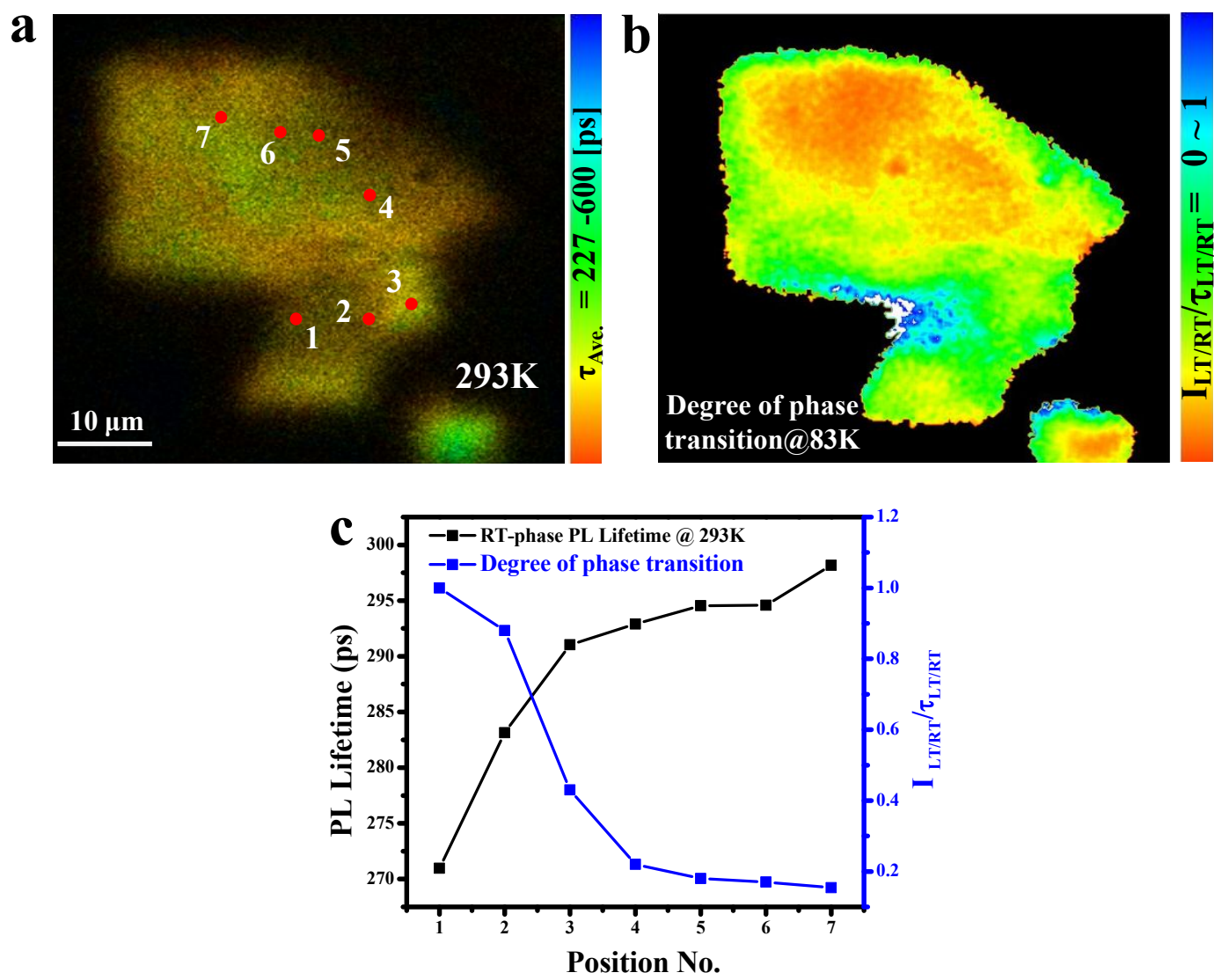

Figure S14. (a) The RT-phase PL lifetime image measured in an individual $\left(\mathrm{n}-\mathrm{C}_{4} \mathrm{H}_{9} \mathrm{NH}_{3}\right)_{2} \mathrm{PbI}_{4} \mathrm{SC}$ at $293 \mathrm{~K}$. (b) The distribution map of the relative completion degree of phase transition at $83 \mathrm{~K}$ calculated according to Eq.(S4), showing a rough inverse correlation relative to the RT-phase PL lifetime image shown in panel a. (c) The comparison of the RT-phase PL lifetimes and the degrees of phase transition from panel $b$ at various positions (denoted by red points) as shown in panel a. 